\section{Bariatric surgery as urate-lowering therapy in severe obesity}

Hyon K Choi, ${ }^{1,2}$ Yuqing Zhang ${ }^{2}$

Gout represents a metabolically driven inflammatory arthropathy, which could be substantially influenced by adiposity and lifestyle risk factors. As such, influenced by the trends in lifestyle factors associated with Westernisation, ${ }^{1}{ }^{2}$ gout prevalence has increased in the last few decades worldwide (eg, 3.9\% of US adults (8.3 million) in $\left.2007-2008^{3}\right){ }^{4}$ The disease burden of gout has been further complicated by a high level of cardiovascular (CV)-metabolic comorbidities (eg, hypertension in $74 \%$; obesity in $53 \%{ }^{5}$ ) and their sequelae (eg, increased future risk of myocardial infarction and premature death ${ }^{67}$ ).

Among many known modifiable risk factors for hyperuricaemia and gout, obesity is one of the strongest, as observed in many prospective cohort studies. ${ }^{8-12}$ To date, few medical interventions, except bariatric surgery, have been effective in the treatment of obesity. Indeed, studies have shown that bariatric surgery not only induces substantial weight loss but also greatly improves key obesity-related CV-metabolic abnormalities and outcomes, ${ }^{13-15}$ including blood pressure, glucose, insulin, triglycerides, highdensity lipoprotein (HDL)-cholesterol, serum uric acid (SUA) levels ${ }^{16}$ and overall mortality. $^{17}$

In their timely study, Dalbeth et al ${ }^{18}$ sought to examine the potential pathogenetic and clinical relevance of the uratelowering benefits of bariatric surgery. Over 1 year of prospective follow-up of 60 individuals with severe obesity (body mass index $(\mathrm{BMI}) \geq 35 \mathrm{~kg} / \mathrm{m}^{2}$ ) and type 2 diabetes, bariatric surgery led to a weight reduction of $34 \mathrm{~kg}$, and proportions of those with SUA levels higher than the urate saturation point and the usual urate-lowering therapy (ULT) target $(\mathrm{SUA}=0.36 \mathrm{mmol} / \mathrm{L})^{19}$ declined by $37 \%$ and $41 \%$, respectively. Among the 12 gout patients included in this study, the proportion of SUA above $0.36 \mathrm{mmol} / \mathrm{L}$ declined from $83 \%(10 / 12)$ at baseline to $33 \%(4 / 12)$ 1 year after surgery; the corresponding

\footnotetext{
${ }^{1}$ Section of Rheumatology, Boston University School of Medicine, Boston, Massachusetts, USA; ${ }^{2}$ Clinical Epidemiology and Training Unit, Boston University School of Medicine, Boston, Massachusetts, USA

Correspondence to Dr Hyon K Choi, Section of Rheumatology and the Clinical Epidemiology Unit, Boston University School of Medicine, 650 Albany Street, Suite 200, Boston, MA 02118, USA; hchoius@bu.edu
}

proportion of ULT use also declined from $75 \%(9 / 12)$ to $33 \%(4 / 12)$. Despite its relatively small sample size, these findings provide additional evidence for the uratelowering benefits of bariatric surgery among obese diabetic patients, and also raise several conceptual and practical issues relevant to the topic.

\section{CAN WEIGHT LOSS INDUCE A CLINICALLY MEANINGFUL URATE REDUCTION?}

Despite some sceptical views on the relevant role of weight loss in the management of hyperuricaemia and gout, the study by Dalbeth et al provides evidence that considerable weight loss can reduce SUA levels in a pathogenetically and clinically meaningful way. This is consistent with the findings from previous studies. ${ }^{13} \quad 15 \quad 20-24$ For example, in the Swedish Obese Subjects Study (total $\mathrm{N}=4047$ ), bariatric surgery decreased SUA levels by $14 \%$ at 2 years $(n=1845)$ and $8 \%$ at 10 years $(n=641)$ compared with their control groups. ${ }^{13}$ Furthermore, bariatric surgery was associated with $78 \%$ and $51 \%$ lower odds of hyperuricaemia at 2 years and 10 years after the surgery, respectively. ${ }^{13}$ In a dietary intervention (as opposed to bariatric surgery) study by Dessein and colleagues, 13 non-diabetic gout patients (mean $\mathrm{BMI}=30.5 \mathrm{~kg} / \mathrm{m}^{2}$ ) received a low-calorie diet over 16 weeks ${ }^{24}$ and achieved a weight loss of $7.7 \mathrm{~kg}$, a SUA reduction of $0.1 \mathrm{mmol} / \mathrm{L}$ (from 0.57 to $0.47 \mathrm{mmol} / \mathrm{L}$ ), and even a reduction in the frequency of monthly gout attacks from 2.1 to 0.6 $(p=0.002)$. Similarly, an analysis based on a lifestyle intervention trial showed that compared with no weight change, the odds of achieving SUA levels of $0.36 \mathrm{mmol} / \mathrm{L}$ for a weight loss of $1-4.9,5-9.9$ and $\geq 10 \mathrm{~kg}$ were $1.43,2.17$ and 3.90 , respectively. ${ }^{23}$ The corresponding ORs of achieving SUA levels of $0.42 \mathrm{mmol} / \mathrm{L}$ were $1.30,1.86$ and 3.66. Consistent findings were observed in a Japanese dietary intervention study. ${ }^{15}$

Dalbeth et al's study was limited to those with diabetes. This is noteworthy because the impact of weight loss on SUA may vary by presence of diabetes, as diabetes and hyperglycaemia are associated with SUA levels lower than in non-diabetic individuals, likely due to the uricosuric effect of glycosuria. ${ }^{25-28}$ For example, a large prospective cohort study $(\mathrm{n}=10000)$ showed that, compared with normal individuals, the prevalence of hyperuricaemia was $63 \%$ lower in men with type 2 diabetes. ${ }^{28}$ This finding can also explain the higher baseline SUA levels in the non-diabetic gout patients $(0.57 \mathrm{mmol} /$ L) of Dessein et al's intervention study ${ }^{24}$ compared with that seen in the diabetic gout patients $(0.41 \mathrm{mmol} / \mathrm{L})$ of Dalbeth et al's study, ${ }^{18}$ despite the considerably higher baseline BMI levels in the latter (30.5 vs $48.5 \mathrm{~kg}$ / $\mathrm{m}^{2}$ ). Furthermore, the underlying mechanism could also explain the discrepancy in the impact of dietary intervention components of the two studies. ${ }^{18} 24$ Regardless, as discussed above, among the 12 gout patients with diabetes, the bariatric surgery resulted in a SUA reduction of $0.08 \mathrm{mmol} / \mathrm{L}$ from the study baseline. This would mean that the large weight loss of $34.0 \mathrm{~kg}$ from bariatric surgery overcame the presumed loss of the uricosuric effects of glycosuria. Correspondingly, it was noteworthy that the level of SUA reduction associated with a weight loss of $34.0 \mathrm{~kg}$ by bariatric surgery among these diabetic patients ${ }^{18}$ was similar to (or possibly smaller than) that achieved by a weight reduction of $7.7 \mathrm{~kg}$ among nondiabetic patients. $^{24}$ Therefore, the SUA impact of this level of weight reduction could be substantially larger among those without diabetes. ${ }^{24}$

\section{POTENTIAL MECHANISMS UNDERLYING THE URATE-LOWERING EFFECTS}

Weight loss is thought to decrease SUA levels primarily by increasing renal excretion of urate and in part by decreasing urate production. ${ }^{27} 29-31$ For example, a previous intervention study found that fractional excretion of uric acid was substantially lower among obese individuals compared with normal controls at baseline (4-5\% vs $11-12 \%$, respectively). ${ }^{15}$ Furthermore, urinary urate excretions were also lower in obese subjects than in controls, suggesting that hyperuricaemia in obese individuals was primarily attributed to impaired renal clearance of uric acid. Importantly, weight loss intervention by diet and exercise resulted in the normalisation of fractional excretion of uric acid among these obese individuals. ${ }^{15}$ Although not addressed in the study by Dalbeth et al, this is likely through declining insulin resistance and insulin levels, which are associated with reduced renal urate excretion and hyperuricaemia. ${ }^{32-34}$ Notably, previous bariatric surgery studies have found a substantial decline in insulin levels and insulin resistance. ${ }^{1635}$

Using linear regression models, Dalbeth et al included potential predictors, 
regardless of temporal ordering, and found that baseline SUA levels, diuretic cessation, glomerular filtration rate (GFR) improvement and sex independently predicted SUA change after bariatric surgery. While this approach might serve a predictive purpose, the causal mechanistic implications of these findings appear limited. This is because these variables represent different time points in causal pathways and thus their effect estimates for potential causal impacts are not directly comparable. ${ }^{36}$ For example, sex and baseline SUA levels should not be mediators in the causal pathway between bariatric surgery and SUA reduction as these variables occur temporally preceding bariatric surgery, whereas the obvious decline in the use of thiazide (from $43 \%$ to $7 \%$ ) is a likely consequence of bariatric surgery, and its effect represents part of the impact of bariatric surgery on SUA levels. Furthermore, the final model failed to find a significant association with weight reduction, which does not appear to make biological sense. Yet, this is expected because the model simultaneously adjusted for downstream mediators such as diuretic use and GFR improvement. To date, various analytic approaches have been developed to partition the total effect of a particular risk factor into plausible causal pathways and to quantify the magnitude of impact of each causal pathway. Employing these methods would clarify the underlying biological mechanisms and quantify the magnitudes of their mediation effects, which in turn can help understand the pathogenetic pathways and potentially improve gout care.

\section{THE BENEFITS OF WEIGHT LOSS WITH REGARD TO COMORBIDITIES OF GOUT}

Beyond urate-lowering benefits in obese hyperuricaemic or gout patients, weight loss improves CV-metabolic-renal abnormalities associated with obesity, ${ }^{24} 3738$ and bariatric surgery may improve survival. ${ }^{17}$ For example, Dessein's dietary intervention study showed significant improvements in total cholesterol, total cholesterol/HDL-C ratio and triglyceride levels. ${ }^{24}$ Similarly, the bariatric surgery-induced weight loss in Dalbeth et al's study was accompanied by an improvement in fasting glucose, HbA1c, GFR, triglycerides and blood pressure. These multiple benefits have been documented by randomised controlled trials (RCTs) that compared bariatric surgery with medical therapy among obese patients with uncontrolled diabetes. ${ }^{37} 38$ For example, an RCT of patients with severe obesity and uncontrolled diabetes showed that at 2 years diabetes remission had occurred in no patients in the medical therapy group versus $75-95 \%$ in the bariatric surgery group. ${ }^{38}$ Furthermore, total cholesterol, triglycerides and HDL cholesterol levels normalised in $27.3 \%, 0 \%$ and $11.1 \%$ of patients in the medical therapy group compared with 100\%, 86-92.3\% and $73-100 \%$ in the bariatric surgery group, respectively. Another RCT of obese patients with uncontrolled diabetes showed that insulin use was $38 \%$ at 12 months in the medical therapy group compared with 4-8\% in the bariatric surgery group. ${ }^{37}$ These multiple CV-metabolic-renal benefits are highly relevant in the holistic management of gout patients as they often have these comorbidities and are at an increased risk of developing related sequelae. Notably, potential CV-metabolic-renal benefits from pharmacological ULT options in gout patients remains largely unknown to date.

In conclusion, despite the lack of a control group and likely underestimation of impact size related to glycosuria-induced uricosuria of diabetic patients, ${ }^{25-27}$ this prospective cohort study adds evidence that bariatric surgery can lead to clinically meaningful SUA target levels and cessation of ULT among gout patients with severe obesity. While the sole purpose of controlling gout would likely not justify bariatric surgery among obese gout patients, if such a surgery is conducted in otherwise indicated patients with gout, it would be reasonable to seek opportunities to withdraw ULT with a proper follow-up plan. Furthermore, the multiple comorbidity and potential survival benefits of this approach and other weight loss measures should be appropriately appreciated in determining their comparative effectiveness with other available ULT options.

Contributors HKC and YZ drafted the manuscript and are guarantors.

Funding HKC is supported by NIH (NIAMS) grants R01-AR056291, R01-AR065944, P60 AR047785 and R21 AR056042. YZ is supported by P60 AR047785.

Competing interests $H K C$ has served on advisory boards for Takeda Pharmaceuticals and Astra-Zeneca Pharmaceuticals, and has received investigator initiated research grants from Takeda Pharmaceuticals and Savient Pharmaceuticals.

Provenance and peer review Commissioned; externally peer reviewed.

To cite Choi HK, Zhang Y. Ann Rheum Dis 2014;73:791-793.

Received 13 January 2014

Accepted 16 February 2014

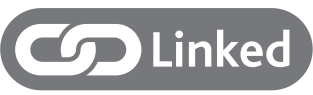

- http://dx.doi.org/10.1136/annrheumdis-2013-203970
Ann Rheum Dis 2014:73:791-793.

doi:10.1136/annrheumdis-2013-204861

\section{REFERENCES}

1 Lawrence RC, Felson DT, Helmick CG, et al. Estimates of the prevalence of arthritis and other rheumatic conditions in the United States: Part II. Arthritis Rheum 2007:58:26-35.

2 Choi HK, Mount DB, Reginato AM. Pathogenesis of gout. Ann Intern Med 2005;143:499-516.

3 Zhu Y, Pandya BJ, Choi HK. Prevalence of gout and hyperuricemia in the US general population. Arthritis Rheum 2011;63:3136-41.

4 Roddy E, Doherty M. Epidemiology of gout. Arthritis Res Ther 2010;12:223.

5 Zhu Y, Pandya BJ, Choi HK. Comorbidities of Gout and Hyperuricemia in the US General PopulationThe National Health and Nutrition Examination Survey 2007-2008. Am J Med 2012;125:679-87.e1.

6 Krishnan E, Baker JF, Furst DE, et al. Gout and the risk of acute myocardial infarction. Arthritis Rheum 2006;54:2688-96.

7 Choi HK, Curhan G. Independent impact of gout on mortality and risk for coronary heart disease. Circulation 2007:116:894-900.

8 Campion EW, Glynn RJ, DeLabry LO. Asymptomatic hyperuricemia. Risks and consequences in the Normative Aging Study. Am J Med 1987;82:421-6.

9 Roubenoff R, Klag MJ, Mead LA, et al. Incidence and risk factors for gout in white men. JAMA 1991:266:3004-7.

10 Choi HK, Atkinson K, Karlson EW, et al. Obesity, weight change, hypertension, diuretic use, and risk of gout in men: the health professionals follow-up study. Arch Intern Med 2005;165:742-8.

11 Bhole V, de Vera M, Rahman MM, et al. Epidemiology of gout in women: fifty-two-year followup of a prospective cohort. Arthritis Rheum 2010;62:1069-76.

12 Rathmann W, Funkhouser E, Dyer AR, et al. Relations of hyperuricemia with the various components of the insulin resistance syndrome in young black and white adults: the CARDIA study. Coronary Artery Risk Development in Young Adults. Ann Epidemiol 1998:8:250-61.

13 Sjostrom L, Lindroos AK, Peltonen M, et al. Lifestyle, diabetes, and cardiovascular risk factors 10 years after bariatric surgery. N Engl J Med 2004;351:2683-93.

14 Gokcel A, Gumurdulu Y, Karakose H, et al. Evaluation of the safety and efficacy of sibutramine, orlistat and metformin in the treatment of obesity. Diabetes Obes Metab 2002;4:49-55.

15 Yamashita S, Matsuzawa Y, Tokunaga K, et al. Studies on the impaired metabolism of uric acid in obese subjects: marked reduction of renal urate excretion and its improvement by a low-calorie diet. Int J Obes 1986;10:255-64.

16 Sjostrom L, Lindroos A-K, Peltonen M, et al. Lifestyle, Diabetes, and Cardiovascular Risk Factors 10 Years after Bariatric Surgery. N Eng/ J Med 2004;351: 2683-93.

17 Sjostrom L, Narbro K, Sjostrom CD, et al. Effects of bariatric surgery on mortality in Swedish obese subjects. N Engl I Med 2007;357:741-52.

18 Dalbeth $\mathrm{N}$, Chen $\mathrm{P}$, White $\mathrm{M}$, et al. Impact of bariatric surgery on serum urate targets in people with morbid obesity and diabetes: a prospective longitudinal study. Ann Rheum Dis 2014;73:797-802.

19 Khanna D, Fitzgerald JD, Khanna PP, et al. 2012 American College of Rheumatology guidelines for management of gout. Part 1: Systematic nonpharmacologic and pharmacologic therapeutic approaches to hyperuricemia. Arthritis Care Res (Hoboken) 2012;64:1431-46.

20 Luyckx FH, Scheen AJ, Desaive C, et al. Effects of gastroplasty on body weight and related biological 
abnormalities in morbid obesity. Diabetes Metab 1998;24:355-61.

21 Pontiroli AE, Pizzocri P, Librenti MC, et al. Laparoscopic adjustable gastric banding for the treatment of morbid (grade 3) obesity and its metabolic complications: a three-year study. I Clin Endocrinol Metab 2002;87:3555-61.

22 Nanji AA, Freeman JB. Rate of weight loss after vertical banded gastroplasty in morbid obesity: relationship to serum lipids and uric acid. Int Surg 1985; 70:323-5.

23 Zhu Y, Zhang Y, Choi HK. The serum urate-lowering impact of weight loss among men with a high cardiovascular risk profile: the Multiple Risk Factor Intervention Trial. Rheumatology (Oxford) 2010;49:2391-9.

24 Dessein PH, Shipton EA, Stanwix AE, et al. Beneficial effects of weight loss associated with moderate calorie/carbohydrate restriction, and increased proportional intake of protein and unsaturated fat on serum urate and lipoprotein levels in gout: a pilot study. Ann Rheum Dis 2000;59:539-43.
25 Cook DG, Shaper AG, Thelle DS, et al. Serum uric acid, serum glucose and diabetes: relationships in a population study. Postgrad Med J 1986;62:1001-6.

26 Choi HK, Ford ES. Haemoglobin A1c, fasting glucose, serum C-peptide and insulin resistance in relation to serum uric acid levels--the Third National Health and Nutrition Examination Survey. Rheumatology (Oxford) 2008:47:713-17.

27 Rodriguez G, Soriano LC, Choi HK. Impact of diabetes against the future risk of developing gout. Ann Rheum Dis 2010;69:2090-4.

28 Herman JB, Goldbourt U. Uric acid and diabetes: observations in a population study. Lancet 1982;2:240-3.

29 Emmerson BT. The management of gout. N Engl J Med 1996;334:445-51.

30 Fam AG. Gout, diet, and the insulin resistance syndrome. J Rheumatol 2002;29:1350-5.

31 Emmerson B. Alteration of urate metabolism by weight reduction. Aust NZ J Med 1973;3:410-12.

32 Emmerson B. Hyperlipidaemia in hyperuricaemia and gout. Ann Rheum Dis 1998;57:509-10.
33 Ter Maaten JC, Voorburg A, Heine RJ, et al. Renal handling of urate and sodium during acute physiological hyperinsulinaemia in healthy subjects. Clin Sci (Lond) 1997:92:51-8.

34 Muscelli E, Natali A, Bianchi S, et al. Effect of insulin on renal sodium and uric acid handling in essential hypertension. Am J Hypertens 1996;9:746-52.

35 Richette P, Poitou C, Garnero P, et al. Benefits of massive weight loss on symptoms, systemic inflammation and cartilage turnover in obese patients with knee osteoarthritis. Ann Rheum Dis 2011;70:139-44.

36 Westreich $D$, Greenland $S$. The table 2 fallacy: presenting and interpreting confounder and modifier coefficients. Am J Epidemiol 2013:177:292-8.

37 Mingrone G, Panunzi S, De Gaetano A, et al. Bariatric surgery versus conventional medical therapy for type 2 diabetes. N Eng/ J Med 2012;366:1577-85.

38 Schauer PR, Kashyap SR, Wolski K, et al. Bariatric surgery versus intensive medical therapy in obese patients with diabetes. N Engl I Med 2012;366:1567-76. 